\title{
Thermally activated flow in soft and hard regions: Getting information on work hardening strain and recovery strain from rate change tests
}

\author{
W. Blum ${ }^{1 *}$, P. Eisenlohr ${ }^{2}$, M. Prell ${ }^{1}$, K. Durst ${ }^{3}$ \\ ${ }^{1}$ Dept. of Materials Science, Institute for General Materials Science, University of Erlangen-Nuremberg, \\ Martensstr. 5, D-91058 Erlangen, Germany \\ ${ }^{2}$ Department of Chemical Engineering and Materials Science, \\ Michigan State University, 428 S. Shaw Lane, East Lansing, MI 48824, U.S.A. \\ ${ }^{3}$ TU Darmstadt, Alarich-Weiss-Str. 2, D-64287 Darmstadt, Germany
}

Received 2 February 2015, received in revised form 27 April 2015, accepted 5 May 2015

\begin{abstract}
The investigation of thermally activated glide processes by stress reduction tests is reviewed. The tests reveal that two mechanisms of inelastic deformation are superimposed, namely dislocation glide connected with work hardening by defect storage and recovery strain related with relaxation of internal stresses and defect recovery. The coupling of the two is proposed to be modeled within the composite model. A new kind of transient testing is proposed that combines the conventional rate change test with an intermediate stress reduction. Its application to nanocrystalline $\mathrm{Ni}$ shows similarity of the main parameters of thermally activated dislocation glide and recovery strain found for conventional pure single and polycrystals: spacings of thermal obstacles in units of free dislocation spacing $\approx 1$, back flow starting after $\approx 20 \%$ stress reduction, similar magnitude of relative contribution and stress dependence of recovery strain.
\end{abstract}

K e y w or d s: thermally activated flow, activation area, composite model, subgrain boundaries, dislocations, recovery

\section{Introduction}

According to classical theory, plastic flow occurs by thermally activated glide of dislocations. The activation energy $Q$ and the activation volume $V$ determine the dependence of the inelastic strain rate $\dot{\varepsilon}_{\text {inel }}$ on temperature $T$ and stress $\sigma$, respectively. $V$ is determined from tests (Fig. 1) where $\sigma$ is varied by a small amount $\Delta \sigma . \dot{\varepsilon}_{\text {inel }}$ responds to the stress reduction in an exponential fashion, i.e. the relative change $\Delta \dot{\varepsilon}_{\text {inel }} / \dot{\varepsilon}_{\text {inel }} \equiv \Delta \ln \dot{\varepsilon}_{\text {inel }}$ is proportional to the resolved shear stress change $\Delta \tau=\Delta \sigma / M$, where $M$ is the crystal orientation factor (Taylor):

$$
\Delta \ln \dot{\varepsilon}_{\text {inel }}=\frac{V}{k_{\mathrm{V}} M k_{\mathrm{B}} T} \Delta \sigma,
$$

where $k_{\mathrm{B}}$ is the Boltzmann constant. This relation holds with $k_{\mathrm{V}}=1$ under the assumption of constant structure in the sense that there is no change except that the stress component driving glide is diminished by $\Delta \tau$ exactly. Obviously, this condition is not easy to guarantee. $\Delta \sigma$ must be small enough and the strain interval $\Delta \varepsilon_{\text {inel }}$ to measure $\dot{\varepsilon}_{\text {inel }} \approx \Delta \varepsilon_{\text {inel }} / \Delta t$ must be as small as possible, but larger than the experimental uncertainty of the strain signal. Even if this is achieved, immediate changes of the true activation volume $V$ may occur:

$$
V=b \Delta x \lambda_{\mathrm{th}}
$$

where $b$ is the Burgers vector length, $\Delta x$ and $\lambda_{\text {th }}$ are the width and the spacing of thermal obstacles, respectively. For discrete, repulsive obstacles changes in $\lambda_{\text {th }}$ result in $k_{\mathrm{V}}=1.5$. To get an idea of the magnitude of $V$ in pure materials one may set $\Delta x \approx b$ and estimate the obstacle spacing by the average spacing of free

*Corresponding author: tel.: +49 9131 63807; e-mail address: wolfgang.blum@ww.uni-erlangen.de 


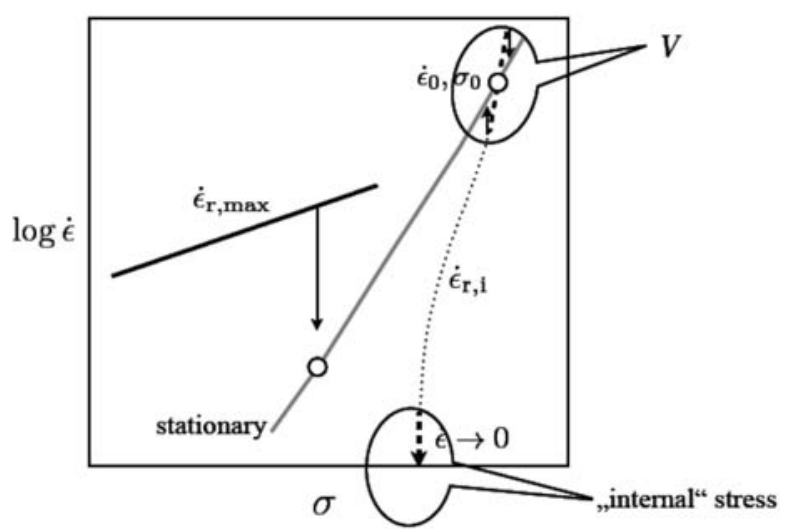

Fig. 1. Scheme of variation of (inelastic) strain rate $\dot{\varepsilon}$ with stress $\sigma$ in change tests at 'constant structure' to determine activation volume $V$ and "internal" stress from first measurable rate response $\dot{\varepsilon}_{\mathrm{r}, \mathrm{i}}$, and relative rate maximum $\dot{\varepsilon}_{\text {r,max }}$ associated with recovery. For simplicity quasistationary behavior is assumed to be established at $\sigma=\sigma_{0}$. After [1].

dislocations $\approx b G / \sigma$. This yields

$$
V_{\mathrm{est}} \approx \frac{G b^{3}}{\sigma_{0}}
$$

where $\sigma_{0}$ is the stress at which the dislocation structure has been formed just before the stress reduction.

There is a large amount of $V$-data in the literature that have been obtained under more or less ideal conditions. ${ }^{1}$ Carefully done experiments generally confirm the estimate of Eq. (3) for pure crystalline materials; for example see data measured and collected for $\mathrm{Al}[2,3], \mathrm{LiF}[4], \mathrm{CaF}_{2}$ [5] and $\mathrm{Ni}$ [6]. This holds up to high homologous temperatures; determination of $V(T)$ from [4] for LiF yields $V$-values within $0.88 \leq V / V_{\text {est }} \leq 0.95$ for $0.68 \leq T / T_{\mathrm{m}} \leq 0.95$. This result suggests that the thermally activated flow of gliding dislocations is the major deformation process up to the melting point $T_{\mathrm{m}}$. However, it does not answer the question for the process controlling the flow of dislocations under given $(\sigma, T)$-conditions.

\section{Implications of recovery strain}

Thermally activated glide of dislocations is complemented by other thermally activated deformation processes like such as those related with recovery of free dislocations and crystallite boundaries $[7,8]$ as well as grain boundary sliding. Due to structural patterning, boundaries of various kinds - from loose cell walls with low misorientation of the cells to high-angle boundaries - are formed during deformation ('straininduced boundaries'). Being strong obstacles [9, 10],

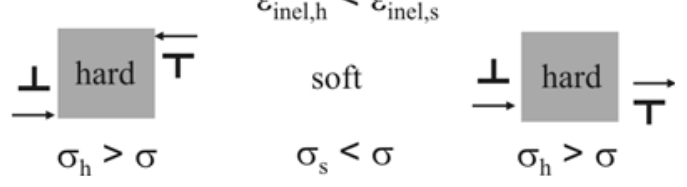

Fig. 2. Composite model of soft and hard regions within a material.

these boundaries are important in controlling the passage of flow from one crystallite to the neighboring one. Under conditions where dynamic recovery can no longer be neglected - and this means during most of the work hardening range - strains associated with recovery must be considered, too.

An obvious case of recovery strain generation is the migration of subgrain boundaries. This is necessary on a more or less local scale for recombination of boundaries via migration of Y-junctions to keep the dynamic balance of formation of new and disappearance of old boundaries in the stationary state. The strain effect connected with boundary migration is directly seen from tilting of the outer surface of those crystal volumes swept by migrating boundaries [1113]; the surface tilts are the result of local shearing of the crystal lattice in the direction prescribed by the acting stress ('migration-shear coupling').

Another obvious positive contribution to recovery strain comes from local dislocation flow in those regions of the material that are relatively hard compared to their environment. The existence of soft and hard regions in any material is accounted for in a simple and illustrative way by Mughrabi's composite model [14, 15] (Fig. 2). Hard regions experience stress concentration due to the easier deformation of their neighborhood. This sets up internal stresses that increase the local stress to $\sigma_{\mathrm{h}}>\sigma$ compared to the applied stress $\sigma$ in the hard regions and (due to mechanical equilibrium) reduce the local stress $\sigma_{\mathrm{s}}<\sigma$ in the soft regions [14]. After a sudden change of an acting stress $\sigma_{0}$ to a reduced value $\sigma$ there are three kinds of recovery processes:

- local back flow in soft regions, reducing the local strain differences and the magnitude of the internal stresses [16] and opening possibilities for recombination (including annihilation) of dislocations at existing crystal defects (other dislocations, crystallite boundaries, ...),

- local forward flow in hard regions qualitatively doing the same,

- migration of boundaries causing strain due to migration-shear coupling, and at the same time caus-

\footnotetext{
1 Some authors have even used the stress exponent for quasi-stationary deformation to determine $V$. This is clearly wrong as quasi-stationary structures, by definition, are not constant, e.g. because of the well-known $\sigma$ dependence of the quasi-stationary dislocation density.
} 
ing boundary recombination and absorption of free dislocations.

These processes can be separated because (i) a stress reduction of a given magnitude $\Delta \sigma$ has a higher relative effect on the low stress $\sigma_{\mathrm{s}}$ than the high stress $\sigma_{\mathrm{h}}$, (ii) $\sigma_{\mathrm{s}}$ is predominantly athermal in nature so that the thermal stress component $\sigma_{\mathrm{s} \text {,th }}$ is but a low fraction of $\sigma$. Thus a relatively small reduction of $\sigma$ is sufficient to stop forward thermally activated glide in the soft regions and drive dislocations backward for a while when $\sigma<\sigma_{\mathrm{s} \text {, ath }}$ at the given high level of the athermal stress component $\sigma_{\mathrm{s} \text {, ath }}$. So small $\sigma$ reductions suffice to effectively suppress thermally activated glide in the soft regions. Therefore, the processes acting in the hard regions under the influence of the concentrated stress $\sigma_{\mathrm{h}}$ are the only ones left and become observable for a while, until they have relaxed again in the transient to the new state corresponding to the new deformation condition, and the majority process of thermally activated flow in the soft regions dominates again. So there is only a limited time window where the recovery strain rate $\dot{\varepsilon}^{-}$is observable. This explains why recovery strain is rarely found. Dedicated search, however, almost always shows recovery strain from single crystals [4] to nanocrystals [17].

Most tests are done under constant imposed rate of length change $i_{\text {mach }}$ imposed by the testing machine. A change of this rate is followed by an extended period of stress relaxation where the plastic deformation rate of the specimen is larger than the elastic rate of length change of specimen plus relevant parts of the testing machine. This relaxation is generally excluded from the data evaluation because $\dot{\varepsilon}_{\text {inel }}$ is not known. However, when the load or the stress is controlled ('creep tests'), recovery strains become easily visible, provided that dynamic recovery is sufficiently active. Clearly, the highest driving force for recovery acts in the quasistationary state. Quasi-stationary means that the dynamic equilibrium of the density of free dislocations has been nearly, but not fully, reached, because some parameters of the structure, for instance those related with the strong obstacles in the form of strain-induced boundaries, are still slowly evolving.

One of the first mentioning of recovery-strain rate $\dot{\varepsilon}^{-}$was in [18] where the connection to subgrain boundaries has already been made. Later, recovery strain was detected also in tests [16] where Al specimens recovered at zero reduced stress. In [19] recovery strain was linked to the composite model. Meanwhile it is well established that recovery strain is a ubiquitous phenomenon. Nevertheless, studies of its kinetics are still rare, although it appears to be of importance under technological (deformation under variable conditions) and fundamental (controlling process) aspects. The present contribution discusses the existing knowledge and extends the concept to nanocrystalline materials.

\section{Typical results for pure materials}

We look at typical results for 'constant structure' deformation kinetics of pure materials with subgrain boundaries of low-angle type established in the quasistationary state, as shown for $\mathrm{Al}$ and $\mathrm{LiF}$ (Figs. 8-19 in [2]). 'Constant structure' is a fuzzy term. Two strain rates of 'constant structure' type must be distinguished (Fig. 1):

$-\dot{\varepsilon}_{\mathrm{r}, \mathrm{i}}$ is the first measurable relative rate after a reduction of stress. Here the structure is as constant as it can possibly be under the given experimental conditions. Limited structural changes can never be excluded because fast ones may occur already in the time interval required for load reduction and measurement of the very first value of $\dot{\varepsilon}_{\text {inel }}=\Delta \varepsilon_{\text {inel }} / \Delta t$ because $\Delta \varepsilon_{\text {inel }}$ cannot be made arbitrarily small, but must exceed the experimental uncertainty of strain measurement.

- $\dot{\varepsilon}_{\mathrm{r}, \mathrm{max}}$ designates the relative rate maxima observed after $\dot{\varepsilon}_{\mathrm{r}, \mathrm{i}}$, if any; here 'constant structure' essentially means constant boundary structure only; changes of the structure of free dislocations have certainly occurred on the way from $\dot{\varepsilon}_{\mathrm{r}, \mathrm{i}}$ to $\dot{\varepsilon}_{\mathrm{r}, \max }[20]$.

$\dot{\varepsilon}_{\mathrm{r}, \mathrm{i}}$ is positive for small reductions of $\sigma$, but turns negative when net back flow starts to dominate for large $\sigma$-reductions. As stated in section $1, \dot{\varepsilon}_{\mathrm{r}, \mathrm{i}}$ is used to determine the activation volume $V$ (upper right corner of Fig. 1). In addition, $\dot{\varepsilon}_{\mathrm{r}, \mathrm{i}}$ has been applied to quantify the athermal flow stress component from the condition $\dot{\varepsilon}_{\mathrm{r}, \mathrm{i}}=0$ (bottom of Fig. 1). Chen et al. [21] have recently reviewed this field. Typically, net back flow sets in for relative stress reductions by about $20 \%$. Then dislocations predominantly flow backwards. Therefore, thermally activated forward dislocation glide of the work hardening type should be blocked for $\sigma \ll$ $\sigma_{0}$. If positive $\dot{\varepsilon}_{\mathrm{r}, \max }$ is still observed under these conditions it should correspond to positive recovery strain and display the maximal recovery-strain rates that can be observed after $\sigma$-reduction. Similar to the case of $\dot{\varepsilon}_{\mathrm{r}, \mathrm{i}}$ the functional relation $\dot{\varepsilon}_{\mathrm{r}, \max }(\sigma)$ contains information about the kinetics of the deformation processes, in particular those in the hard regions where recovery is concentrated. The slope of the $\dot{\varepsilon}_{\mathrm{r}, \max }$-line (Fig. 1) may correspond to the thermal obstacle spacings in the hard regions. Here the dislocation spacings are tighter than in the soft regions. For instance, in the quasistationary low-angle boundaries the spacings can be estimated as $s=b / \theta$ where $\theta$ is the subgrain misorientation. In typical cases $s$-values fall by a factor of 10 and more below the spacings $\delta$ in the soft regions [2]. So one might expect a correspondingly lower slope of $\dot{\varepsilon}_{\mathrm{r}, \max }(\sigma)$ compared to $\dot{\varepsilon}_{\mathrm{r}, \mathrm{i}}(\sigma)$. However, the ratio of slopes is distinctly less than 10 .

In discussing this result one has to note that according to the composite model (compare Fig. 2) in the form presented in $[19,22]$ the (inelastic) strain rate 
$\dot{\varepsilon}$ consists of three major components:

$$
\dot{\varepsilon}=f_{\mathrm{s}} \dot{\varepsilon}_{\mathrm{s}}\left(\sigma_{\mathrm{s}}\right)+f_{\mathrm{h}} \dot{\varepsilon}_{\mathrm{h}}\left(\sigma_{\mathrm{h}}\right)+\dot{\varepsilon}_{\mathrm{migr}},
$$

where $f_{\mathrm{s}}$ and $f_{\mathrm{h}}$ are the volume fractions of soft (subscript $\mathrm{s}$ ) and hard (subscript $\mathrm{h}$ ) regions, respectively, $\dot{\varepsilon}_{\mathrm{s}}$ and $\dot{\varepsilon}_{\mathrm{h}}$ are the local inelastic strain rates due to glide of free dislocations, and $\dot{\varepsilon}_{\text {migr }}$ is the strain rate due to recovery of boundary area by migration. Passage through the hard regions tends to cause recovery of free dislocations by reaction with the boundaries or free dislocations of opposite sign waiting at the other side of the boundaries. The recovery strain rate with positive sign follows as

$$
\dot{\varepsilon}^{-}=f_{\mathrm{h}} \dot{\varepsilon}_{\mathrm{h}}\left(\sigma_{\mathrm{h}}\right)+\dot{\varepsilon}_{\text {migr }} .
$$

The quantitative meaning of the slope of the $\dot{\varepsilon}_{\mathrm{r}, \max }(\sigma)$ line is not clear at present:

- Superposition of the two recovery strain terms in Eq. (5) leads to an effective activation volume lying in between those of $\dot{\varepsilon}_{\mathrm{h}}$ and $\dot{\varepsilon}_{\text {migr }}$.

- Recovery in the time period where local back flow overcompensates forward recovery strain causes an increasing amount of recovery of internal stresses that leads to reduction of $\sigma_{\mathrm{h}}$ exceeding the stress reduction. It was shown in [5] that the observed $\dot{\varepsilon}_{\mathrm{r}, \max (\sigma)}$ lies between the boundaries found from the assumption that the internal forward stress in the hard regions remains constant or relaxes in proportion to $\sigma$.

- The width of the thermal obstacles in the hard regions increases with decreasing $\sigma_{\mathrm{h}}$ making deformation increasingly more difficult.

So far the kinetics of recovery strain has not been understood. Dedicated tests concentrating on this issue might be a big help in achieving better understanding of the strength of materials in cases where recovery is important (not only at high $T$, but also at high $\sigma$ in strong materials).

\section{Transient behavior of nanocrystalline Ni after stress reduction and rate change}

So far we have treated single or coarse-grained polycrystals. Nanocrystalline (nc) materials are special in that their boundary spacing is distinctly less than the quasistationary boundary spacing $w_{\mathrm{qs}}$ in the former materials even though the strength of the material is so high that high normalized flow stresses result. The question therefore is whether such nc materials differ in their response to stress changes from the "normal" materials. Two similar compression tests were done on nc Ni proving the reproducibility of the results. In these tests a fast stress reduction was combined with a large reduction of imposed compression rate by a factor of 1000 . The intention of this test procedure was to

- save enough of the high driving force for dynamic recovery introduced during the fast initial deformation to make recovery strain visible,

- limit the duration of the transient to the new quasi-stationary state by keeping the imposed strain rate constant.

Special care was taken to quantify and remove the elastic components of the strain signal to get the inelastic strain of the specimen continuously over the whole test without gaps during fast stress changes. Figure 3 displays the results of one of the tests. In the short initial period of fast deformation (light gray) the flow stress rises to a level of $1890 \mathrm{MPa}$. The fast stress drop to $1540 \mathrm{MPa}$ takes $16 \mathrm{~s}$ (Fig. 3b). In this short period the inelastic strain rate $\dot{\varepsilon}_{\text {inel }}$ falls continuously by a factor of about 50 . In the long subsequent period of low total (elastic plus inelastic) strain rate $\dot{\varepsilon}_{\text {tot }}=1 \times 10^{-6} \mathrm{~s}^{-1}$ (dark gray) the inelastic strain rate $\dot{\varepsilon}_{\text {inel }}$ gradually falls further until it has become equal to $\dot{\varepsilon}_{\text {tot }}$. As $\dot{\varepsilon}_{\text {inel }}$ is always larger than $\dot{\varepsilon}_{\text {tot }}$, the stress $\sigma$ is gradually relaxing down to $\approx 1000 \mathrm{MPa}$. From this value the stress increases again toward the new quasistationary level without reaching saturation. The last period of the test is fast again at $\dot{\varepsilon}_{\text {tot }}=1 \times 10^{-3} \mathrm{~s}^{-1}$ and a quasi-stationary level of flow stress is finally reached (Fig. 3a).

During the slow deformation significant recovery must have occurred. This follows from the fast work hardening when the fast deformation is resumed again. The recovered defects are quickly replaced by the currents of free dislocations being held up at the boundaries. The work hardening path is qualitatively similar to the initial one (Fig. 3a,c), but differs quantitatively because the predeformation has raised the defect density compared to the undeformed state.

The opposite, namely softening, has to occur in the periods of reduced $\sigma$ and $\dot{\varepsilon}_{\text {tot }}$. This is the case. The first response to the decline of stress is the deceleration of glide of free dislocations near the starting stress of $1890 \mathrm{MPa}$. The dash-dotted line shows the slope expected from Eq. (3). It is consistent with thermally activated glide of dislocations just as in coarse-grained materials. However, this process should come to a stop at some point when the dislocations have gotten stuck and the rate of inelastic deformation of the work hardening type should remain zero until recovery has suficiently reduced the high density of defects stored during the preceding fast deformation. But the contrary is observed. The ongoing recovery is accompanied by inelastic deformation at relatively high rate. So this rate is not of the work hardening type, but of the recovery type as described above. As the delayed back flow of dislocations ceases, there is even net softening as the recovery strain is no longer masked by concurrent back flow. This is obvious from the significant decrease in flow stress by $300 \mathrm{MPa}$ at nearly constant 



Fig. 3. Compression test on nc Ni at constant total strain rate $\dot{\varepsilon}_{\text {tot }}=1 \times 10^{-3} \mathrm{~s}^{-1}$ and $373 \mathrm{~K}$ with intermediate reduction of stress followed by deformation at low total strain rate $\dot{\varepsilon}_{\text {tot }}=1 \times 10^{-6} \mathrm{~s}^{-1}$; the stress $\sigma$ is shown as a function of (a) $\varepsilon_{\text {inel }}$, (b) time $t$, and (c) in relation to the inelastic strain rate $\dot{\varepsilon}_{\text {inel }} . \dot{\varepsilon}_{\text {r,max }}$ from normalized curve for nc Ni in Fig. 5 of [17].

rate of inelastic deformation $\approx 1.3 \times 10^{-5} \mathrm{~s}^{-1}$ (Fig. 3). The measured rates are close to the maximum recov- ery strain rates $\dot{\varepsilon}_{\text {r,max }}(\sigma)$ found in [17] from sudden stress changes just after the delayed ('anelastic') back flow in nc Ni at room temperature. In this case recovery was proven by in situ-observation of X-ray line widths. So we have reason to assume that the same processes of recovery strain, probably by boundary migration [23], occur here, too.

This result explains why attempts to determine an 'internal stress' (athermal stress component) for thermally activated glide have been unsatisfactory [24]: in the transient from high to low rate the material switches from deformation of the work hardening type by thermally activated glide to deformation of the recovery type. The athermal stress component for the work hardening strain rate cannot be derived from such a test. The same holds for stress reduction tests (stress dip tests) to determine an internal stress' (Fig. 1) from onset of back flow (see e.g. [25]). This is not the point where thermally activated glide ceases, but the point where delayed back flow occurs at the same rate as recovery strain. This implies that the 'internal stress' for thermally activated glide is systematically determined too low. It seems possible that many of the inconsistencies listed in [24] vanish when the existence of recovery strain is taken into proper account. This may lead to a revival of the stress reduction techniques aiming at determining the athermal stress component for glide of the work hardening type.

\section{Summary}

Tests with large relative stress reductions are known to yield information on the different mechanisms contributing to deformation. Most of the inelastic strain usually results from dislocation glide accompanied by storage of defects and work hardening. However, there is also another mechanism of deformation that on the one hand is well known, but still appears to be underestimated so far. That is the strain associated with recovery of internal stresses and straininduced defects, in particular free dislocations and internal crystal boundaries. With increasing flow stress the share that recovery strain has of the total inelastic strain increases. Stress reduction tests make the recovery strain visible the better the more the work hardening strain gets suppressed. The recovery strain is of particular importance as it controls the motion of free dislocations and in this sense may be regarded as the controlling mechanism.

We have shown that the determination of the $\dot{\varepsilon}_{\text {inel }}(\sigma)$ relation at (nearly) constant structure is a means to separate the mechanisms of work hardening strain and recovery strain from each other. This separation is a prerequisite to study their intrinsic kinetics. It is suggested that work hardening strain is concentrated in the soft regions of the material while recov- 
ery strain is concentrated in the hard regions. From this point of view the composite model of inelastic deformation appears to be ideal as a framework to bring the different mechanisms of deformation together and model their interaction by setup of internal stresses.

A disadvantage of tests with large stress reductions is that deformation at the low stress level gradually becomes too slow to be measured in reasonable time spans when the recovery activity falls from the initial high level due to predeformation to the new, low quasi-stationary one. The present work demonstrates (for the first time as far as the authors are aware) that this disadvantage of testing the response to large stress reductions, as required for the separation of mechanisms, can easily be overcome by continuing deformation not at constant stress, but at constant low imposed total deformation rate. The choice of this rate is independent of the degree of stress reduction, delivers an additional, independent test parameter, and limits the duration of the tests ad libitum.

If the inelastic strain rate is measured not only after the changes of the deformation parameters, but also during the changes by subtracting the elastic response of specimen and machine from the total one, the parameters of thermally activated glide in the soft regions can be determined. It is found that nanocrystalline Ni shows the same pattern of deformation that is found for single or conventional polycrystalline pure materials. The activation volume yields thermal obstacle spacings that lie in the order of the expected dislocation spacing $b G / \sigma$ in the soft regions. For large stress reductions, forward deformation continues by recovery strain similar to what has been found for single and conventional polycrystals. This suggests that the basic mechanisms of deformation of pure crystalline materials remain essentially the same during grain refinement down into the nanocrystalline regime with grain sizes in the order of 100 atomic spacings.

\section{Acknowledgement}

This paper is dedicated to Professor Pavel Lukáč on the occasion of his 80 th birthday.

\section{References}

[1] Blum, W.: In: Proceedings of ISPMA13. Prague, Charles University in Prague 2014. doi:10.13140/ 2.1.2498.1760

[2] Blum, W.: High-Temperature Deformation and Creep of Crystalline Solids. In: Plastic Deformation and Fracture of Materials. Ed.: Mughrabi, H. Vol. 6 of Materials Science and Technology. Eds.: Cahn, R. W., Haasen, P., Kramer, E. J., Weinheim, VCH Verlagsgesellschaft 1993.
[3] Blum, W., Pöllmann, T., Hofmann, U., Weidinger, P.: Phys. Stat. Sol. A, 157, 1996, p. 329. doi:10.1002/pssa.2211570215

[4] Müller, W., Biberger, M., Blum, W.: Philos. Mag. A, 66, 1992, p. 717. doi:10.1080/01418619208201586

[5] Mekala, S., Eisenlohr, P., Blum, W.: Philos. Mag., 91, 2011, p. 908. doi:10.1080/14786435.2010.535324

[6] Li, Y. J., Mueller, J., Höppel, H., Göken, M., Blum, W.: Acta Mater., 55, 2007, p. 5708. doi:10.1016/j.actamat.2007.06.036

[7] Blum, W., Nix, W. D.: Res. Mechanica Letters, 1, 1981, p. 235.

[8] Blum, W.: Scripta Metall., 18, 1984, p. 1383. doi: 10.1016/0036-9748(84)90370-3

[9] Liu, B., Raabe, D., Eisenlohr, P., Roters, F., Arsenlis, A., Hommes, G.: Acta Materialia, 59, 2011, p. 7125. doi:10.1016/i.actamat.2011.07.067

[10] Liu, B., Eisenlohr, P., Roters, F., Raabe, D.: Acta Materialia, 60, 2012, p. 5380. doi:10.1016/i.actamat.2012.05.002

[11] Exell, S. F., Warrington, D. H.: Philos. Mag. A, 26, 1972, p. 1121.

[12] Molodov, D., Ivanov, V., Gottstein, G.: Acta Materialia, 55, 2007. p. 1843. doi:10.1016/j.actamat.2006.10.045

[13] Gorkaya, T., Burlet, T., Molodov, D. A., Gottstein, G.: Scripta Materialia, 63, 2010, p. 633. doi:10.1016/j.scriptamat.2010.05.040

[14] Mughrabi, H.: In: Proceedings of 5th Int. Conf. on the Strength of Metals and Alloys. ICSMA 5. Eds.: Haasen, P., Gerold, V., Kostorz, G. Oxford, Pergamon Press 1980, p. 1615.

[15] Mughrabi, H.: Acta Metall., 31, 1983, p. 1367. doi:10.1016/0001-6160(83)90007-X

[16] Hasegawa, T., Yakou, T., Kocks, U.: Acta Metall., 30, 1982, p. 235.

[17] Sun, Z., Petegem, S. V., Cervellino, A., Durst, K., Blum, W., Swygenhoven, H. V.: Acta Materialia, 91, 2015, p. 91. doi:10.1016/i.actamat.2015.03.033

[18] Blum, W., Hausselt, J., König, G.: Acta Metall., 24, 1976, p. 293. doi:10.1016/0001-6160(76)90003-1

[19] Blum, W., Rosen, A., Cegielska, A., Martin, J. L.: Acta Metall., 37, 1989, p. 2439.

[20] Hausselt, J., Blum, W.: Acta Metall., 24, 1976, p. 1027. doi:10.1016/0001-6160(76)90133-4

[21] Chen, B., Flewitt, P. E. J., Cocks, A. C. F., Smith, D. J.: International Materials Reviews, 60, 2015, p. 1. doi:10.1179/1743280414Y.0000000041

[22] Vogler, S., Blum, W.: Creep and Fracture of Engineering Materials and Structures. Eds.: Wilshire, B., Evans, R. London, The Institute of Metals 1993.

[23] Lohmiller, J., Grewer, M., Braun, C., Kobler, A., Kübel, C., Schüler, K., Honkimäkig, V., Hahn, H., Kraft, O., Birringer, R., Gruber, P. A.: Acta Materialia, 65, 2014, p. 295. $\underline{\text { doi:10.1016/i.actamat.2013.10.071 }}$ 
[24] Chen, B., Hu, J., Flewitt, P., Smith, D., Cocks, A., Zhang, S.: Acta Materialia, 67, 2014, p. 207. doi:10.1016/j.actamat.2013.12.027
[25] Blum, W., Finkel, A.: Acta Metall., 30, 1982, p. 1705. 